Leiva Arcas, A.; Sánchez Pato, A.; Martínez Patiño, M.J. (2021) Impact Analysis of ADO Plan in the Spanish Olympic Results. Revista Internacional de Medicina y Ciencias de la Actividad Física

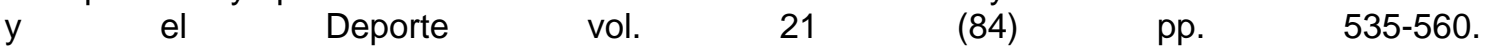
Http://cdeporte.rediris.es/revista/revista83/artanalisis1274.htm

DOI: https://doi.org/10.15366/rimcafd2021.83.008

\title{
ORIGINAL
}

\section{ANÁLISIS DEL IMPACTO DEL PLAN ADO EN LOS RESULTADOS OLÍMPICOS ESPAÑOLES}

\section{IMPACT ANALYSIS OF ADO PLAN IN THE SPANISH OLYMPIC RESULTS}

\author{
Leiva Arcas, A. ${ }^{1}$; Sánchez Pato, A. ${ }^{1}$ y Martínez Patiño, M.J. ${ }^{2}$ \\ ${ }^{1}$ Profesor, Centro de Estudios Olímpicos de la Universidad Católica de Murcia. Murcia (España) \\ aleiva@ucam.edu \\ ${ }^{1}$ Decano, Centro de Estudios Olímpicos de la Universidad Católica de Murcia. Murcia (España). \\ apato@ucam.edu \\ 2 Profesora, Facultad de Ciencias de la Educación y del Deporte de la Universidad de Vigo. \\ Pontevedra (España) mipatino@uvigo.es
}

AGRADECIMIENTOS: Ios autores agradecen al Comité Olímpico Español el acceso a los datos sobre la participación olímpica española, y al Dr. Juan García Manso por su ayuda y consejo en el tratamiento de los mismos.

Código UNESCO I UNESCO Code: 5599 Otras especialidades históricas: Historia del Deporte / Others historical specialties: History of Sport.

Clasificación del Consejo de Europa / Council of Europe Classification: 7 Historia del Deporte / History of Sport

Recibido 25 de julio de 2019 Received July 25, 2019

Aceptado 20 de octubre de 2019 Accepted October 20, 2019

\section{RESUMEN}

En el presente artículo se analizan las políticas deportivas españolas y su influencia en el rendimiento olímpico, poniendo el foco de atención en el programa de Apoyo a los Deportistas Olímpicos (Plan ADO). Se describe posteriormente cómo se han distribuido las becas ADO y cuál ha sido la evolución de la participación olímpica española bajo este programa de apoyo a la tecnificación y profesionalización del deporte de élite nacional. El estudio continúa con el análisis del rendimiento de los deportistas españoles en las distintas ediciones de los Juegos dentro del desarrollo de este programa, con especial atención al periodo 2005-2016, enfatizando la relación entre la aportación económica global del Plan ADO en forma de becas y los resultados obtenidos. Como conclusión, se constata que el Plan ADO ha sido uno de los 
factores claves de la transformación deportiva en España y de los éxitos internacionales desde Barcelona 1992.

PALABRAS CLAVE: Plan ADO, Olimpismo, Juegos Olímpicos, Becas ADO.

\begin{abstract}
In this article, Spanish sports policies and their influence on Olympic performance are analyzed, focusing on the program of Support for Olympic Sportsmen (ADO Plan, by the acronym in Spanish). It is described below how the ADO scholarships have been distributed and what has been the evolution of the Spanish Olympic participation under this program that supports the technification and professionalization of the national elite sport. The study continues with the analysis of the performance of Spanish athletes in the different editions of the Games within the development of this program, with special attention to the 2005-2016 period, emphasizing the relationship between the global economic contribution of the ADO Plan through scholarships and the results obtained. In conclusion, it can be seen that the ADO Plan has been one of the key factors in sports transformation in Spain and international successes since Barcelona 1992.
\end{abstract}

KEY WORDS: ADO Plan, Olympism, Olympic Games, ADO Scholarships.

\title{
INTRODUCCIÓN
}

A pesar de que los Juegos Olímpicos modernos fueron concebidos por el Barón Pierre de Coubertin como un proyecto de unión y concordia para la manifestación de la grandeza deportiva (Pérez-Aragón y Gallardo-Pérez, 2017), actualmente muchos países invierten importantes cantidades de dinero en sus mejores atletas para que sean más competitivos y consigan alzarse con el triunfo en las distintas pruebas deportivas (Johnson y Ali, 2004; Humphreys et al., 2018; Haut et al., 2017).

Desde que el deporte de alto nivel alcanza la transcendencia actual, muchos han sido los intentos de indagar en los aspectos más importantes en los que se sustenta el éxito en las competiciones internacionales, muy especialmente en los Juegos Olímpicos (JJOO) (Castejón et al., 1973; Kiviaho y Makelä, 1978; Douyin, 1988; Greenleaf, Gould y Diefen, 2001; Morton, 2002; Conzelmann y Nagel, 2003; Gibbons et al., 2003; Bernard y Megan, 2004; Green y Houlihan, 2005; De Bosscher et al., 2008; Funahashi et al., 2015; Robles-Rodríguez et al., 2019).

Sin duda, son muchas las variables que pueden influir en los triunfos (medallas o títulos) que consiga un país en los JJOO, Campeonatos del Mundo (CM) o grandes competiciones internacionales en las que se enfrenta al resto de países; dejando a un lado a los análisis que evaluaron el deporte del antiguo bloque socialista (Krüger, 1984; Riordan, 1989; Semotiouk, 1990), donde otros factores pueden haber sido determinantes. 
En esa línea, Gómez et al., (2011) los analizan y unifica en nueve grupos diferentes: financiación pública o privada; desarrollo de deportistas de elite; nivel de práctica deportiva del país; iniciación y participación deportiva a nivel estatal; búsqueda, identificación y desarrollo de talentos deportivos; profesionalización de los deportistas mediante el apoyo económico, técnicos especialistas, además de incentivos por logros deportivos conseguidos; desarrollo de las instalaciones deportivas de y para la elite; formación y profesionalización de entrenadores; participación en competiciones internacionales e investigación y tecnología aplicada al alto rendimiento. No obstante, y como bien señalan De Bosscher et al. (2009), por muchas variables que introduzcamos en la ecuación, la posibilidad de dar con la fórmula mágica es prácticamente imposible de lograr.

En cualquier caso, lo que sí es una realidad es que las políticas deportivas bien diseñadas son la base fundamental sobre la que se sustenta el logro de altos resultados deportivos en el deporte de élite (Green y Houlihan, 2005; De Bosscher, et al., 2006; Bergsgard et al., 2007; Houlihan y Green, 2008; BarkerRuchti et al., 2018). En opinión de algunos autores (De Bosscher et al., 2008; De Bosscher et al., 2015; Reiche, 2016) tampoco en este punto estamos cerca de encontrar el modelo ideal.

En España, las políticas de apoyo gubernamental al deporte olímpico han sido escasas. Durante la etapa de la dictadura Franquista (1936-1975), el apoyo al olimpismo estuvo marcado por una clara intencionalidad política, enfocado a un lavado de cara del Régimen en el plano de la diplomacia internacional más que al desarrollo de una estructura de apoyo estable al deporte profesional (Santacama, 2011). Ello conllevó que muchos deportistas olímpicos se vieran obligados a sufragar con sus propios medios los gastos inherentes a su formación y tecnificación deportiva (Calle-Molina y Martínez-Gorroño, 2019). Con la transición política acaecida en nuestro país tras la muerte de Franco, en otoño de 1975, los cambios sociales se fueron produciendo de forma vertiginosa y, sorprendentemente, de forma pacífica. Las autoridades públicas, y en particular los municipios tras las elecciones municipales de 1979, asumieron la responsabilidad de acercar la práctica deportiva a todos los sectores de la población (Puig et al., 2010). Sin embargo, la responsabilidad en el deporte de élite recayó sobre la Dirección General de Educación Física y Deportes (Real Decreto 596/1977, abril de 1977), para sustituir a la antigua Delegación Nacional de Deportes y, principalmente en las Federaciones Deportivas Nacionales y el Comité Olímpico Español (COE). Debemos recordar que la Dirección General de Educación Física y Deportes poco después pasó a denominarse Centro Superior de Educación Física y Deportes (Decreto 1119/1977, mayo de 1977) y, finalmente, Consejo Superior de Deportes (CSD) (Decreto 2258/1977 del 27 de agosto de 1977).

En la vigente Constitución Española de 1978 ya se recalcaba la importancia del deporte al incluir en su Artículo 43.3 que "Los poderes públicos fomentarán la educación sanitaria, la educación física y el deporte. Asimismo, facilitarán la adecuada utilización del ocio".

Durante esta etapa, el máximo responsable político de estas instituciones fue Benito Castejón y Paz-Pardo. Militar de carrera y abogado, presenta un largo y 
productivo historial como dirigente deportivo que entendemos necesario destacar por lo que supuso al crear las bases del futuro Plan ADO. Fue delegado Nacional de Educación Física y Deportes desde septiembre de 1976 hasta abril de 1977 y, posteriormente, Secretario de Estado para el Deporte (25 de enero de 1980) y Presidente del Comité Olímpico Español desde esa fecha hasta 1980 (12 de mayo de 1980). Debemos tener en cuenta que desde el final de la Guerra Civil española ambas instituciones estaban unidas y bajo la tutela estatal, continuando en esta anómala situación hasta 1984 con el nombramiento de Alfonso de Borbón y Dampierre, duque de Cádiz y primo del rey Juan Carlos I, como $12^{\circ}$ Presidente del COE.

En el primer punto, España, desde la Constitución de 1977 (Título I, capítulo III, art. 43.3), ya había incorporado al deporte como una obligación del Estado, lo que queda reforzado por la Ley General de la Cultura Física y del Deporte (Ley 13/1980 de 31 de marzo) y, especialmente, por la Ley del Deporte de 1990 (Ley 10/1990). En esta reforma legislativa es donde se comienza a dar forma legal a la figura del deportista de Alto Nivel. La ley especifica que el deporte de Alto Nivel es un aspecto de interés para el Estado que incrementa los niveles de práctica deportiva a la vez que permite crear la Reserva Deportiva necesaria con la que poder afrontar con éxito los compromisos deportivos internacionales en los que participan los deportistas, equipos y selecciones nacionales. Es en esta Ley donde en su Título $V$ (Artículos 48 y 49), se regula la organización y funciones del COE en la España democrática.

Es en 1995 (Real Decreto 1856/1995) cuando se establece de forma legal quién puede ser considerado deportista de Alto Nivel. Para ello, se crean la Comisión de Evaluación del Deporte de Alto Nivel y las Subcomisiones Técnicas de Seguimiento y Planificación. No obstante, este primer documento fue modificado en 2007 (Real Decreto 971/2007) cuando se incluyen aspectos relevantes que regulan los beneficios que deben recibir este sector de deportistas.

Otro momento clave en la historia del deporte español es la designación de Barcelona como sede de la XXV edición de los JJOO de verano. Era el cuarto intento que realizaba nuestro país para conseguir tan prestigioso nombramiento (Barcelona: 1924; 1936 y 1940 y Madrid: 1972). La iniciativa para que Barcelona se presentara como candidata a los Juegos Olímpicos de 1992 partió del alcalde Narcís Serra, quien el 30 de junio de 1981 llevó al pleno del Consistorio la propuesta, la cual fue liderada por Pasqual Maragall (alcalde de la ciudad entre 1982 y 1997). Su triunfo con 47 votos sobre las ciudades de París (29 votos), Belgrado, Brisbane, Birmingham y Ámsterdam fue anunciada, y previamente apoyada, por Juan Antonio Samaranch (Presidente del COI), el 17 de octubre de 1986 en la ciudad suiza de Lausana.

\section{DESARROLLO DE LA INVESTIGACIÓN}

La investigación desarrollada en este artículo busca satisfacer un doble objetivo. En primer lugar, se pretende analizar la estructura del Plan ADO, desde su génesis hasta nuestros días, analizando las principales líneas maestras que han guiado las políticas de apoyo al deporte de élite en nuestro país y promovidas desde este organismo. Seguidamente, se busca describir la influencia que este 
Plan ha tenido en la realidad deportiva española - expresado en el número de becas concedidas - confrontando con las cifras de participación y resultados de los deportistas olímpicos españoles, con especial atención al periodo comprendido entre 2005 y 2016. Del mismo modo, el estudio se centrará en el impacto económico que este programa ha tenido en el deporte de alto nivel nacional. Los datos utilizados han sido recogidos a través de diversas fuentes consultadas por los autores en el Consejo Superior de Deportes y en el Comité Olímpico Español

\section{El Programa de Apoyo a los Deportistas Olímpicos (ADO)}

La implantación de modelos deportivos eficientes no es posible sin disponer del adecuado soporte jurídico y la suficiente dotación económica con que poder desarrollarlos. Esto es especialmente complejo en un modelo de país como es España que, desde un punto de vista competencial (Estado de las Autonomías), presenta serias dificultades a la hora de evaluar e interpretar por qué y cómo surgen deportistas del más alto nivel internacional. No olvidemos que, principalmente, el CSD, el COE y las Federaciones Nacionales, pero también las Comunidades Autónomas, los Cabildos y Diputaciones e, incluso, algunos Ayuntamientos, participan de una u otra manera en el proceso de formación o apoyo a los deportistas de élite.

La difícil tarea de capitanear la transformación deportiva desde el final de la Dictadura durante los primeros años de la transición recayó en Benito Castejón Paz, primer presidente del CSD de 1977 a 1980. Licenciado en Derecho y militar perteneciente al Cuerpo Jurídico de Aviación, fue uno de los primeros políticos que trataron de racionalizar y evaluar el deporte de Alto Rendimiento, así como crear estructuras deportivas (Centros de Iniciación Técnicos, Deportiva, Centros de Perfeccionamiento y Centros de Alto Rendimiento) que permitieran conseguir un deporte de élite español capaz de competir dignamente en el contexto internacional. Todas esas ideas quedan reflejadas en la obra Rationalising Sports Policies (1973), en colaboración con Juan de Dios García-Martínez y José Rodríguez Carballada. El objetivo principal de este estudio, presentado en Estrasburgo ante el Consejo de Europa, fue diseñar un modelo para racionalizar las políticas deportivas gubernamentales, definiendo los conceptos básicos que deberían ser inherentes a cualquier política deportiva con independencia de la diversidad que caracteriza al deporte moderno. En la segunda y la tercera parte de su trabajo desarrolla un modelo matemático con el que poder optimizar y racionalizar la toma de decisiones en la política deportiva. Una de las características más relevantes de este trabajo es el contenido, bien documentado y desarrollados, de diferentes instrumentos estadísticos para implementar el modelo que proponían los autores.

Creado el 24 de diciembre de 1987 por la Asociación de Deportes Olímpicos (Comité Olímpico Español, Consejo Superior de Deporte y Radio Televisión Española), el Plan de Apoyo al Deportista Olímpico se erigió como una institución sin ánimo de lucro. Su principal objetivo es el apoyo, desarrollo y promoción de los deportistas nacionales de Alto Nivel que pudieran formar parte del equipo olímpico español que participara en los Juegos Olímpicos de Barclona'92 y posteriores. Su organigrama y funcionalidad ha ido evolucionando a través de 
los años adaptándose a las necesidades, cuando no los problemas, que surgían en cada momento. Uno de los momentos más importantes, se produjo en septiembre de 2005 cuando se creó el Consorcio de Empresas ADO, que incluía al Ministerio de Economía y Hacienda, Comité Olímpico Español, Asociación de Deportes Olímpicos y Consejo Superior de Deportes.

Por su transcendencia y eficacia se ha ido prorrogando en cada ciclo olímpico hasta los JJOO de Tokio 2020 (8 ciclos en los que se incluyen los JJOO de verano e invierno de cada olimpiada). Así, por ejemplo, el actual ciclo olímpico (Rio de janeiro 2016 a Tokio 2020), incluye los programas de preparación para los JJOO de invierno a celebrar en Pyeongchang (2018) y Pekín (2022) y los de verano de Tokio 2020.

Ello supone crear estrategias vinculadas a aspectos tan diversos como:

- Desarrollar y promocionar lo máximo posible las diferentes modalidades deportivas olímpicas.

- Detectar los talentos deportivos más destacados que sean capaces de rendir a alto nivel y lograr la clasificación para unos Juegos.

- Preparar los deportistas seleccionados de forma eficiente y con los mejores medios técnicos y materiales posibles.

- Buscar recursos económicos (públicos y privados) para sufragar la asistencia a competiciones y sustentar las carreras profesionales de deportistas y entrenadores.

- Velar por el adecuado desarrollo del programa creado para cada Olimpiada.

Desde 2004 existe paralelamente el Programa ADOP que da apoyo a los deportistas con discapacidad en su preparación para los Juegos Paralímpicos.

El Plan ADO dispone de dos órganos de representación (Asamblea General y Junta Directiva) en los que participan representantes de cada uno de los socios constituyentes. La Asamblea General se reúne ordinariamente dos veces por año, y la Junta Directiva está presidida por dos copresidentes, un vicepresidente, un secretario y los gestores divididos en dos comisiones, la Técnica y la Económica. Los copresidentes se rotan anualmente en el cargo, correspondiendo en los años impares al presidente del CSD y en los años pares al presidente del COE.

Incluye diferentes tipos de programas de ayudas que se establecen es cada ciclo olímpico, con criterios, recursos y reglamentaciones específicas. Estos programas son de tres tipos:

1. Becas a los deportistas más destacados y con posibilidades de conseguir resultados relevantes durante los correspondientes JJOO. Para ello cuenten con los medios económicos adecuados para una correcta preparación. 
2. Incentivos a los técnicos responsables de la preparación de los deportistas seleccionados. Incluyen a los entrenadores personales (pertenezcan o no a los cuadros técnicos de la correspondiente federación), que reciben una beca similar a la del deportista También incluye a las personas que tienen una responsabilidad directa en la preparación de estos deportistas.

3. Planes Especiales que complementan la preparación de los deportistas contemplados en el programa. Estos planes son coordinados con las federaciones deportivas correspondientes e incluyen, entre otros, aspectos tales como: adquisición de material específico y de última generación; promoción del deporte femenino; contratación de técnicos, concentraciones permanentes o temporales; asistencia a competiciones de alto nivel; programas de investigación tecnológica; seguimiento internacional de los potenciales rivales; $y$, control y apoyo psicológico, biomédico y de recuperación.

Quizás el programa más específico es el de Becas a los deportistas. Es oportuno destacar que los recursos totales pueden variar cada temporada en función de los recursos aportados por las instituciones participantes y los socios contribuyentes. La magnitud de las Becas y los criterios de obtención también pueden variar, previo acuerdo de la Junta Directiva en función de diferentes criterios técnicos (resultados deportivos obtenidos). Se distinguen tres tipos de Becas que responden a las características de cada modalidad deportiva:

- Grupo I: Atletas que practican en deportes individuales, incluidos los relevos (natación y atletismo) y los deportistas de equipos con un número inferior a cuatro componentes (dobles de bádminton, dobles de tenis de mesa, dúo de sincronizada, persecución por equipos en ciclismo de pista, etc.).

- Grupo II: Deportistas que realizan pruebas de equipos en los que participan simultáneamente más de cuatro deportistas (equipo sincronizada, conjunto de gimnasia rítmica y remo a 8).

- Grupo III: Participantes en competiciones de equipos colectivos (baloncesto, balonmano, fútbol, hockey sobre hielo, hockey sobre hierba, voleibol y waterpolo).

Para estos deportistas existen 5 tipos de becas (M: Beca Medalla; D: Beca Diploma; R: B: Beca Resultado; E: Beca Especial; y C: Beca Clasificación), cuya cuantía está condicionada por dos factores: el grupo al que pertenece la modalidad deportiva del atleta y los resultados obtenidos en las competiciones internacionales más importantes del año anterior (Juegos Olímpicos-JJOO; Campeonatos del Mundo-CM, Campeonatos de Europa-CE o Ranking Mundial$\mathrm{RM})$. La asignación de las becas M y D está compuesta por una parte fija y una parte variable que corresponden con el 60 y el $40 \%$ respectivamente de la cuantía asignada para cada nivel. 
En los Grupos I y II, salvo algunas consideraciones concretas que no consideramos oportunas para este trabajo, las Becas Medalla (M), son de tres niveles según el lugar que ocupe el deportista en el podio de los JJOO o los CM (M1: Oro; M2: Plata; M3: Bronce). Este tipo de beca no se asigna en los Campeonatos de Europa. Las becas Resultado son de cinco niveles (D1: $4^{\circ}$ Puesto; D2: $5^{\circ}$ Puesto; D3: $6^{\circ}$ Puesto; D4: $7^{\circ}$ Puesto y D5: Puesto $8^{\circ}$ en JJOO o CM) También se entregan Becas Diploma para los mejor clasificados en CE (D1: $1^{\circ}$ Puesto; D2: $2^{\circ}$ o $3^{\circ}$ Puesto; D3: $4^{\circ}$ Puesto; D4: 50 Puesto y D5: Puesto $6^{\circ}$ ). Las Becas Resultado cambian ligeramente en función de que el deportista pertenezca a los Grupos I o II. En el primer caso se entrega a los deportistas que ocupen los puestos $9^{\circ}$ a $12^{\circ}$ de los JJOO o $\mathrm{CM}$ o se clasifiquen entre los 10 primeros del ranking mundial de la especialidad. Los del G-II obtienen Beca $R$ cuando se clasifican entre los puestos $9^{\circ}$ o $10^{\circ}$ de los $\mathrm{JJOO}$ o el CM.

En el Grupo III, los criterios de obtención de Becas cambian ostensiblemente por las propias características del deporte. Las Becas M1, M2 y M3 se les asigna al $1^{\circ}, 2^{\circ}$ y $3^{\circ}$ de los $\mathrm{JJOO}$ o CM, Mientras que las Becas $D$ incluyen a los resultados de los CE (D1: $4^{\circ} \mathrm{JJOO}$ y CM o a $1^{\circ}, 2^{\circ}$ o $3^{\circ}$ de CE; D2: $5^{\circ}$ o $6^{\circ}$ de los JJOO y $\mathrm{CM}$ o $4^{\circ}$ del CE; $\mathrm{D}: 5^{\circ}$ o $\left.6^{\circ} \mathrm{CE}\right)$. En este Grupo, el monto total de la Beca lo reciben las respectivas Federaciones, quienes posteriormente se encargan de determinar qué deportistas obtienen la ayuda y su cuantía.

Para una adecuada motivación y garantizar la mayor transparencia posible, ADO publica anualmente los listados con la cantidad monetaria que conseguiría el deportista en cada caso. Así, un deportista que se corona campeón del Mundo en el año anterior, recibirá una beca mayor que otro deportista que haya quedado en la tercera posición, o que otro que haya sido campeón de Europa. De esta forma, para el actual ciclo Olímpico (Tokio 2020) las cuantías quedan reflejadas en las tablas 1 a 3 (GI), 2 (GII) y 3(GIII). 
Rev.int.med.cienc.act.fís.deporte - vol. 21 - número 83 - ISSN: 1577-0354

Tabla 1. Becas del Plan ADO Grupo I (cantidad en euros).

\begin{tabular}{|c|c|c|c|c|c|}
\hline DEPORTE & BECA & $\begin{array}{l}\text { PARTE } \\
\text { FIJA }\end{array}$ & $\begin{array}{l}\text { PARTE } \\
\text { VARIABLE }\end{array}$ & $\begin{array}{l}\text { CUANTÍA } \\
\text { TOTAL }\end{array}$ & CRITERIO \\
\hline Atletismo & M1 & 36.000 & 24.000 & 60.000 & $1^{\circ} \mathrm{JJOO}$ \\
\hline Bádminton & & & & & $1^{\circ} \mathrm{CM}$ \\
\hline Baloncesto (3x 3) & M2 & 30.300 & 20.200 & 50.500 & $2^{\circ} \mathrm{JJOO}$ \\
\hline Boxeo & & & & & $2^{\circ} \mathrm{CM}$ \\
\hline Ciclismo & M3 & 27.000 & 18.000 & 45.000 & $3^{\circ} \mathrm{JJOO}$ \\
\hline Deportes de invierno & & & & & $3^{\circ} \mathrm{CM}$ \\
\hline $\begin{array}{l}\text { Deportes de Hielo (sin } \\
\text { Hockey) }\end{array}$ & D1 & 20.400 & 13.600 & 34.000 & $4^{\circ} \mathrm{JJOO}$ \\
\hline Escalada & & & & & $4^{\circ} \mathrm{CM}$ \\
\hline Esgrima & & & & & $1^{\circ} \mathrm{CE}$ \\
\hline $\begin{array}{l}\text { Gimnasia } \\
\text { (Individulal) }\end{array}$ & D2 & 18.300 & 12.200 & 30.500 & $5^{\circ} \mathrm{JjOO}$ \\
\hline Gimnasia Rítmica (Individual) & & & & & $5^{\mathrm{a}} \mathrm{CM}$ \\
\hline Golf & & & & & $2^{\circ}-3^{\circ} \mathrm{CE}$ \\
\hline Halterofilia & D3 & 16.200 & 10.800 & 27.000 & $6^{\circ} \mathrm{JJOO}$ \\
\hline Hípica & & & & & $6^{\mathrm{a}} \mathrm{CM}$ \\
\hline Judo & & & & & $4^{\circ} \mathrm{CE}$ \\
\hline Karate & D4 & 14.400 & 9.600 & 24.000 & $7^{\circ} \mathrm{JJOO}$ \\
\hline Lucha & & & & & $7^{\mathrm{a}} \mathrm{CM}$ \\
\hline $\begin{array}{l}\text { Natación (sin sincronizada ni } \\
\text { waterpolo) }\end{array}$ & & & & & $5^{\circ} \mathrm{CE}$ \\
\hline Pentatlón Moderno & D5 & 12.300 & 8.200 & 20.500 & $8^{\circ} \mathrm{JJOO}$ \\
\hline Piragüismo & & & & & $8^{\mathrm{a}} \mathrm{CM}$ \\
\hline Remo (excelto 8+) & & & & & $6^{\circ} \mathrm{CE}$ \\
\hline Surf & $\mathrm{R}$ & 10.000 & & 10.000 & $\begin{array}{l}9^{\circ}-12^{\circ} \\
\mathrm{JJOO}\end{array}$ \\
\hline Skateboarding & & & & & $9^{\circ}-12^{\circ} \mathrm{CM}$ \\
\hline Taekwondo & & & & & 1 a $10 \mathrm{RM}$ \\
\hline $\begin{array}{l}\text { Tenis } \\
\text { Tenis de Mesa }\end{array}$ & $E$ & 10.000 & & 10.000 & $\begin{array}{l}\text { Excepto } \\
\text { años JjOO }\end{array}$ \\
\hline Tiro con Arco & $\mathrm{C}$ & 8.000 & & 10.000 & Clasificados \\
\hline Tiro Olímpico & & & & & sin Beca \\
\hline Triatlón & & & & & JJOO \\
\hline Vela & & & & & 2018-2020 \\
\hline Voley Playa & & & & & \\
\hline
\end{tabular}

Fuente: elaboración propia 
Rev.int.med.cienc.act.fís.deporte - vol. 21 - número 83 - ISSN: 1577-0354

Tabla 2. Becas del Plan ADO Grupo II (cantidad en euros).

\begin{tabular}{|c|c|c|c|c|c|}
\hline DEPORTE & BECA & PARTE FIJA & PARTE VARIABLE & $\begin{array}{l}\text { CUANTÍA } \\
\text { TOTAL }\end{array}$ & CRITERIO \\
\hline $\begin{array}{l}\text { Gimnasia Rítmica } \\
\text { (Conjunto) } \\
\\
\text { Natación } \\
\text { Sincronizada } \\
\text { (Conjunto) } \\
\text { Remo 8+ }\end{array}$ & $\begin{array}{l}\text { M1 } \\
\text { M2 } \\
\text { M3 }\end{array}$ & $\begin{array}{l}24.000 \\
20.220 \\
18.000 \\
13.620 \\
12.240 \\
10.800\end{array}$ & $\begin{array}{l}16.000 \\
13.480 \\
12.000 \\
9.000 \\
8.160 \\
7.200\end{array}$ & $\begin{array}{l}40.000 \\
33.750 \\
30.000 \\
22.700 \\
20.400 \\
18.000 \\
16.000 \\
13.700 \\
8.000 \\
8.000 \\
8.000\end{array}$ & $\begin{array}{l}1^{\circ} \mathrm{JJOO} \\
1^{\circ} \mathrm{CM} \\
2^{\circ} \mathrm{JJOO} \\
2^{\circ} \mathrm{CM} \\
3^{\circ} \mathrm{JJOO} \\
3^{\circ} \mathrm{CM} \\
4^{\circ} \mathrm{JJOO} \\
4^{\circ} \mathrm{CM} \\
1^{\circ} \mathrm{CE} \\
5^{\circ} \mathrm{JJOO} \\
5^{\mathrm{a}} \mathrm{CM} \\
2^{\circ}-3^{\circ} \mathrm{CE} \\
6^{\circ} \mathrm{JJOO} \\
6^{\mathrm{a}} \mathrm{CM} \\
4^{\circ} \mathrm{CE} \\
7^{\circ} \mathrm{JJOO} \\
7^{\mathrm{a}} \mathrm{CM} \\
5^{\circ} \mathrm{CE} \\
8^{\circ} \mathrm{JJOO} \\
8^{\mathrm{a}} \mathrm{CM} \\
6^{\circ} \mathrm{CE} \\
9^{\circ}-10^{\circ} \\
\text { JJOO } \\
9^{\circ}-10^{\circ} \mathrm{CM} \\
\text { Excepto } \\
\text { Año JJOO } \\
\text { Clasificados } \\
\text { JJOO } \\
\mathrm{CM}\end{array}$ \\
\hline
\end{tabular}

Fuente: elaboración propia 
Tabla 3. Becas del Plan ADO Grupo III (cantidad en euros).

\begin{tabular}{|c|c|c|c|c|c|}
\hline DEPORTE & BECA & PARTE FIJA & PARTE VARIABLE & $\begin{array}{l}\text { CUANTÍA } \\
\text { TOTAL }\end{array}$ & CRITERIO \\
\hline $\begin{array}{l}\text { Baloncesto } \\
\text { Balonmano } \\
\text { Béisbol } \\
\text { Fútbol } \\
\text { Hockey } \\
\text { Hockey Hielo } \\
\text { Rugby } \\
\text { Softbol } \\
\text { Voleibol Sala } \\
\text { Waterpolo }\end{array}$ & $\begin{array}{l}\text { M3 } \\
\text { D1 }\end{array}$ & $\begin{array}{l}10.200 \\
9.000 \\
8.000 \\
8.000\end{array}$ & $\begin{array}{l}12.000 \\
10.000 \\
8.800 \\
7.400\end{array}$ & $\begin{array}{l}30.000 \\
25.000 \\
22.000 \\
18.500 \\
17.000 \\
15.000 \\
8.000 \\
8.000\end{array}$ & $\begin{array}{l}1^{\circ} \mathrm{JJOO} \\
1^{\circ} \mathrm{CM} \\
2^{\circ} \mathrm{JJOO} \\
2^{\circ} \mathrm{CM} \\
3^{\circ} \mathrm{JJOO} \\
3^{\circ} \mathrm{CM} \\
4^{\circ} \mathrm{JJOO} \\
4^{\circ} \mathrm{CM} \\
1^{\circ} \mathrm{CE} \\
5^{\circ} \mathrm{JJOO} \\
5^{\mathrm{a}} \mathrm{CM} \\
2^{\circ}-3^{\circ} \mathrm{CE} \\
6^{\circ} \mathrm{JJOO} \\
6^{\mathrm{a}} \mathrm{CM} \\
4^{\circ} \mathrm{CE} \\
\text { Excepto } \\
\text { Año } \\
\text { Olímpico } \\
\text { Clasificados } \\
\text { para } \\
\mathrm{JJOO} \text { y CM }\end{array}$ \\
\hline
\end{tabular}

Fuente: elaboración propia

Evaluación e impacto del programa ADO.

Si los programas de Alto Rendimiento desarrollados por el Gobierno de España durante la transición fueron una revolución respecto a su enfoque y la implementación de recursos, no es hasta que aparece el programa ADO cuando España comienza a ocupar un lugar importante en el deporte internacional. Ciertamente sería una injusticia plantear que ADO haya sido el único factor determinante, pero sin duda es uno de los aspectos con mayor peso en la transformación del deporte de élite nacional. Una de las formas más sencillas y útiles de comprobar su efectividad es realizar un análisis del número de deportistas clasificados para unos Juegos y la evaluación de los resultados obtenidos por los mismos. El número de deportistas beneficiados no ha disminuido. 
Tabla. 4. Evolución de las becas del Plan ADO (2005-16).

\begin{tabular}{|c|c|c|c|c|}
\hline \multirow[b]{2}{*}{ AÑO } & \multicolumn{4}{|c|}{ CICLO OLÍMPICO 2005-2008 } \\
\hline & $\begin{array}{l}\text { Becas ADO } \\
\text { Totales }\end{array}$ & $\begin{array}{l}\text { Becas ADO } \\
\text { Hombres }\end{array}$ & $\begin{array}{l}\text { Becas ADO } \\
\text { Mujeres }\end{array}$ & $\begin{array}{l}\text { Ratio } \\
\text { H:M }\end{array}$ \\
\hline 2005 & 414 & 238 & 174 & 1.37 \\
\hline 2006 & 434 & 264 & 168 & 1.57 \\
\hline 2007 & 374 & 213 & 161 & 1.32 \\
\hline \multirow[t]{5}{*}{2008} & 368 & 202 & 163 & 1.24 \\
\hline & \multicolumn{4}{|c|}{ DEPORTISTAS PARTICIPANTES EN PEKÍN 2008} \\
\hline & 285 & 164 & 121 & 1.36 \\
\hline & \multicolumn{4}{|c|}{ CICLO OLÍMPICO 2009-2012 } \\
\hline & $\begin{array}{l}\text { Becas ADO } \\
\text { Totales }\end{array}$ & $\begin{array}{l}\text { Becas ADO } \\
\text { Hombres }\end{array}$ & $\begin{array}{l}\text { Becas ADO } \\
\text { Mujeres }\end{array}$ & $\begin{array}{l}\text { Ratio } \\
\text { H:M }\end{array}$ \\
\hline 2009 & 404 & 224 & 182 & 1.23 \\
\hline 2010 & 355 & 182 & 171 & 1.06 \\
\hline 2011 & 356 & 193 & 166 & 1.16 \\
\hline 2012 & 330 & 184 & 141 & 1.30 \\
\hline \multirow[b]{5}{*}{2013} & \multicolumn{4}{|c|}{ DEPORTISTAS PARTICIPANTES EN LONDRES 2012} \\
\hline & 281 & 170 & 111 & 1.53 \\
\hline & \multicolumn{4}{|c|}{ CICLO OLÍMPICO 2012-2016 } \\
\hline & $\begin{array}{l}\text { Becas ADO } \\
\text { Totales }\end{array}$ & $\begin{array}{l}\text { Becas ADO } \\
\text { Hombres }\end{array}$ & $\begin{array}{l}\text { Becas ADO } \\
\text { Mujeres }\end{array}$ & $\begin{array}{l}\text { Ratio } \\
\text { H:M }\end{array}$ \\
\hline & 193 & 114 & 78 & 1.46 \\
\hline 2014 & 326 & 145 & 180 & 0.81 \\
\hline 2015 & 399 & 197 & 200 & 0.99 \\
\hline \multirow[t]{3}{*}{2016} & 439 & 245 & 199 & 1.23 \\
\hline & \multicolumn{4}{|c|}{ DEPORTISTAS PARTICIPANTES EN RÍO 2016} \\
\hline & 309 & 165 & 144 & 1.15 \\
\hline
\end{tabular}

Fuente: elaboración propia

En la Tabla 4 podemos observar la evolución de distribución de becas concedidas por el Plan ADO entre 2005 y 2016. Llaman la atención dos aspectos: en primer lugar, el descenso de becas concedidas desde 2005 alcanzando la cifra más baja en 2013 con sólo 193 deportistas gratificados. Estas variaciones se deben fundamentalmente al aumento o reducción de la inversión privada por parte de las empresas patrocinadoras, cuestión que se tratará más adelante. En segundo lugar, podemos observar que los deportistas masculinos reciben un mayor número de becas que las deportistas femeninas, salvo en los años 2014 y 2015 donde la ratio se invierte a favor de las mujeres; y en los años 2010 y 2011 donde este valor queda cerca de 1. Llamativamente, será en los JJOO de estos dos ciclos, Londres 2012 y Rio 2016, donde las mujeres obtengan por primera vez más medallas que los varones y donde las cifras de participación por sexo alcancen cifras cercanas a la paridad (ver Tablas 5 y 10). 
Otro aspecto interesante es que, a pesar de la oscilación en el numero de becas adjudicadas, el número de deportistas varones que han obtenido plaza para estos tres Juegos apenas ha variado $(164,170$ y 165), mientras que el número de mujeres ha crecido sensiblemente, al calificar 144 para Rio de Janeiro, por 111 de Londres y 121 de Pekín. Este incremento en la participación femenina coincide, como hemos comentado, con el único ciclo olímpico en el que las mujeres han superado por primera vez a los hombres en becas ADO.

Evolución de la participación y resultados de la delegación olímpica española

Desde la celebración de los primeros Juegos Olímpicos en Atenas en 1896 hasta la actualidad, un total de 156.462 atletas (115.771 hombres y 41.001 mujeres) han participado en unos Juegos durante 120 años de historia del Movimiento Olímpico contemporáneo (Tabla 5). España ha participado, hasta los JJOO de Rio de Janeiro de 2016 con un total de 3.649 deportistas (2.721 han sido hombres y 928 mujeres), lo que supone el 2,3\% del total de los atletas olímpicos (Leiva-Arcas y Sánchez-Pato, 2019). 
Tabla 5. Evolución de la participación olímpica española (1986-2016).

\begin{tabular}{|c|c|c|c|c|c|c|c|c|c|}
\hline \multirow[t]{2}{*}{ JjOO } & \multicolumn{3}{|c|}{ Total deportistas olímpicos } & \multicolumn{2}{|c|}{$\begin{array}{l}\text { Hombres } \\
\text { España }\end{array}$} & \multicolumn{2}{|c|}{$\begin{array}{l}\text { Mujeres } \\
\text { España }\end{array}$} & \multicolumn{2}{|c|}{$\begin{array}{l}\text { Totales } \\
\text { España }\end{array}$} \\
\hline & Hombres & Mujeres & Totales & $\mathbf{N}^{\circ}$ & $\%$ & $\mathbf{N}^{\circ}$ & $\%$ & $\mathrm{~N}^{\circ}$ & $\%$ \\
\hline Atenas'96 & 241 & 0 & 241 & & & & & & \\
\hline París‘00 & 975 & 22 & 997 & 3 & 0,31 & & & 3 & 0,30 \\
\hline San Luis' 04 & 645 & 6 & 651 & & & & & & \\
\hline Londres'08 & 1971 & 37 & 2008 & & & & & & \\
\hline Estocolmo'12 & 2359 & 48 & 2047 & & & & & & \\
\hline Amberes'20 & 2561 & 65 & 2626 & 56 & 2,19 & & & 56 & 2,13 \\
\hline París'24 & 2954 & 135 & 3089 & 98 & 3,32 & 4 & 2,96 & 102 & 3,30 \\
\hline Amsterdam'28 & 2606 & 277 & 2883 & 81 & 3,11 & & & 81 & 2,81 \\
\hline Los Ángeles'32 & 1206 & 126 & 1332 & 6 & 0,50 & & & 6 & 0,45 \\
\hline Berlín’36 & 3632 & 331 & 3963 & & & & & & \\
\hline Londres'48 & 3714 & 390 & 4104 & 63 & 1,70 & & & 63 & 1,54 \\
\hline Helsinki'52 & 4436 & 519 & 4955 & 27 & 0,61 & & & 27 & 0,54 \\
\hline Melbourne'56 & 2938 & 376 & 3314 & & & & & & \\
\hline Roma'60 & 4727 & 611 & 5338 & 134 & 2,83 & 11 & 1,80 & 145 & 2,72 \\
\hline Tokio'64 & 4473 & 678 & 5151 & 47 & 1,05 & 3 & 0,44 & 50 & 0,97 \\
\hline México'68 & 4735 & 781 & 5516 & 101 & 2,13 & 2 & 0,26 & 103 & 1,87 \\
\hline Munich'72 & 6065 & 1058 & 7173 & 118 & 1,95 & 5 & 0,47 & 123 & 1,71 \\
\hline Montreal'76 & 4824 & 1260 & 6084 & 103 & 2,14 & 11 & 0,87 & 114 & 1,87 \\
\hline Moscú'80 & 4064 & 1115 & 5179 & 147 & 3,62 & 9 & 0,81 & 156 & 3,01 \\
\hline Los Ángeles'84 & 5263 & 1566 & 6829 & 164 & 3,12 & 16 & 1,02 & 180 & 2,64 \\
\hline Seúl'88 & 6197 & 2194 & 8391 & 187 & 3,02 & 29 & 1,32 & 216 & 2,57 \\
\hline Barcelona'92 & 6652 & 2704 & 9356 & 296 & 4,45 & 125 & 4,62 & 421 & 4,50 \\
\hline Atlanta'96 & 6806 & 3512 & 10318 & 196 & 2,88 & 93 & 2,65 & 289 & 2,80 \\
\hline Sídney' 00 & 6582 & 4069 & 10651 & 218 & 3,31 & 105 & 2,58 & 323 & 3,03 \\
\hline Atenas'04 & 6296 & 4329 & 10625 & 177 & 2,81 & 139 & 3,21 & 316 & 2,97 \\
\hline Pekín’08 & 6526 & 4802 & 11328 & 164 & 2,51 & 121 & 2,52 & 285 & 2,52 \\
\hline Londres 2012 & 6053 & 4836 & 10889 & 170 & 2,81 & 111 & 2,30 & 281 & 2,58 \\
\hline Rio 2016 & 6270 & 5154 & 11424 & 165 & 2,63 & 144 & 2,79 & 309 & 2,70 \\
\hline Total: & 115.771 & 41.001 & 156.462 & 2.721 & 2,35 & 928 & 2,26 & 3649 & 2,33 \\
\hline
\end{tabular}

Fuente: elaboración propia

Representando a España, han participado históricamente más hombres (2.721), que mujeres (928). No obstante, en cuanto al peso porcentual de los atletas españoles en el total de los deportistas olímpicos a nivel mundial, observamos un porcentaje casi similar: $2,35 \%$ de todos los atletas olímpicos masculinos de la Era Moderna han sido españoles, por un 2,26\% de los femeninos.

En cuanto a los resultados obtenidos por los atletas españoles, en este punto, hay que distinguir dos etapas cronológicas bien diferenciadas: antes y después de los JJOO de Barcelona 92. En materia de resultados, la celebración de estos Juegos tuvo una relevancia determinante en el posterior devenir de los éxitos del equipo olímpico español. España ha obtenido a lo largo de su historia un total de 150 medallas (44 de Oro; 65 de Plata; 41 de Bronce). Esto sitúa a nuestro país 
en el puesto vigesimoctavo del ranking de países que han obtenido más medallas en la Historia de los JJOO.

En la actualidad, tras los Juegos de Rio 2016, España ocupa la decimocuarta posición del ranking de países, mejorando significativamente el puesto ocupado en los JJOO de Seúl'88 (25 junto a Finlandia) e incluso la mejor posición de todos los tiempos ( $20^{\circ}$ en Moscú 1980 y Los Ángeles 1984) donde se vivieron los dos grandes bloqueos sufridos pos los JJOO (Moscú: 66 países y Los Ángeles 14 países), y sin tener en cuenta la posición en el medallero de París 1900, unos Juegos en los que aún se discute la oficiliadidad de muchas de sus pruebas. Sólo se han logrado finalizar entre los 15 mejores países clasificados en tres ocasiones: en Barcelona 1992 (6ª), Atlanta 1996 (13ª) y Pekín 2008 (14ª). Debe ser tenido en cuenta que esta posición mejora la que nos correspondería teóricamente por número de habitantes $\left(30^{\circ} 46.653 .000\right.$ habitantes) e iguala a la que nos correspondería por potencial económico (13 $13^{\circ} 1.437 .047$ millones de $\mathrm{PIB})$, que son dos de los factores más importantes que actúan sobre el éxito deportivo de los países.

Una forma interesante de evaluar los resultados obtenidos en los diferentes JJOO es a partir de los puestos que ocuparon los deportistas participantes. Debemos tener en cuenta que el COI no ofrece una clasificación por Comités Olímpicos Nacionales ya que según la Carta Olímpica los Juegos Olímpicos son una competición entre deportistas y no entre países. No obstante, existe un sistema de puntuación (Global, Hombres y Mujeres) donde se valora de mayor a menor las medallas de oro, plata y bronce más diplomas conseguidos. En ocasiones las puntuaciones de las medallas son sobrevaloradas y en otras ocasiones nos. Tampoco existe un criterio unánime ni oficial sobre el número de puestos valorados, los cuales oscilan entre el $6^{\circ}$ y $8^{\circ}$ puesto (Sergeyev, 2015). Por este motivo, con fines explicativos, tomaremos para este trabajo un criterio de clasificación basado en el siguiente sistema de puntuación de sobrevaloración del medallero: Oro 12 puntos; Plata 9 puntos; Bronce 7 puntos; $4^{\circ}$ Puestos 5 puntos; $5^{\mathrm{a}}$ Puesto 4 puntos; $6^{\mathrm{a}}$ Puesto 3 puntos; $7^{\circ}$ Puesto 2 puntos; $8^{\circ}$ Puesto 1 punto, tal y como establece el Comité Olímpico Internacional

Tabla 6. Españoles entre los ocho primeros clasificados en JJOO (1992-2016).

\begin{tabular}{|l|l|l|l|l|l|l|l|l|l|}
\hline JjO0 & O & $\mathbf{P}$ & $\mathbf{B}$ & $\mathbf{4}^{\mathbf{0}}$ & $\mathbf{5}^{\mathbf{0}}$ & $\mathbf{6}^{\mathbf{0}}$ & $\mathbf{7}^{\mathbf{0}}$ & $\mathbf{8}^{\mathbf{0}}$ & Puntos \\
\hline Barcelona'92 & 13 & 7 & 2 & 5 & 9 & 6 & 3 & 7 & 320 \\
Atlanta'06 & 5 & 6 & 6 & 4 & 9 & 8 & 10 & 4 & 260 \\
Sydney'00 & 3 & 3 & 5 & 10 & 12 & 7 & 3 & 6 & 185 \\
Atenas'04 & 3 & 11 & 6 & 6 & 14 & 3 & 14 & 8 & 322 \\
Pekín'08 & 5 & 11 & 3 & 5 & 12 & 5 & 7 & 6 & 288 \\
Londres'12 & 4 & 10 & 4 & 8 & 6 & 7 & 5 & 4 & 265 \\
Rio'16 & 7 & 4 & 6 & 6 & 15 & 4 & 7 & 7 & 285 \\
\hline
\end{tabular}

Fuente: elaboración propia 
De acuerdo a la puntuación lograda se detectan dos ediciones claves en las que la delegación española obtuvo los mejores resultados en unos JJOO. El primero, como era de esperar, corresponde a los JJOO de Barcelona 1992 y el segundo fueron los JJOO de Atenas 2004.

En cualquier modalidad deportiva, competir como local constituye una ventaja que ha sido ampliamente estudiada y documentada, pero no siempre bien comprendida (Jones, 2013). Son muchos los factores que pueden influir en este fenómeno, y pueden variar significativamente según el país donde se celebre el evento, las características del deporte y la influencia de factores externos al sistema de puntuación. De acuerdo al análisis de estudios previos, Legaz-Arrese et al., (2013), se plantean cinco causas principales que permiten explicar las razones que justifican esta ventaja: la presencia de aficionados, la familiaridad con el entorno, la ausencia de viajes, la aplicación de las normas y el sentimiento de territorialidad. Estos factores podrían convertirse en elementos condicionantes del comportamiento de deportistas, entrenadores y árbitros.

El repunte del deporte español en los Juegos de Atenas se sustenta en el elevado número de diplomas conseguidos por la delegación española (51 Diplomas), el mayor de la historia, y el decisivo peso que tuvo el equipo femenino (6 Medallas y 25 Diplomas).

\section{La incidencia del Plan ADO en los resultados del equipo olímpico español}

Para España, los JJOO de Barcelona 1992 supusieron un verdadero punto de inflexión. Solamente en esta edición se obtuvieron 22 preseas, 13 de ellas de oro, casi las mismas que en el resto de citas celebradas hasta la fecha. Desde entonces, el olimpismo español ha mejorado con creces sus resultados olímpicos, sin llegar a repetirse el éxito de Barcelona, pero con un desarrollo constante que ha provocado que desde 1992 hasta 2016 se hayan obtenido 123 medallas, es decir, el 82\% del total, 39 de las cuales han sido de oro. Si bien es cierto que estos resultados se han obtenido sobre una base de participación que se ha incrementado notablemente, ya que, en estos últimos siete JJOO se concentra el $61 \%$ de la participación total (2.224 atletas). Observando la Tabla 8, comprobamos que hasta 1992, los deportistas españoles se habían alzado con un total de 27 medallas (todas ellas conseguidas por atletas varones), los que supone únicamente un $18 \%$ del total de metales obtenidos, si bien es cierto que en términos porcentuales la participación registrada hasta aquel momento fue de 1425 atletas, es decir, el 39\% del total de olímpicos españoles. 
Tabla 8. Resultados del equipo olímpico español en los JjOO de la era moderna antes y después de Barcelona 1992.

\begin{tabular}{|c|c|c|c|c|c|c|}
\hline JJOO & Atletas & Oros & Platas & Bronces & Total & $\begin{array}{l}\text { Posición } \\
\text { Medallero }\end{array}$ \\
\hline \multicolumn{7}{|l|}{ Atenas'96 } \\
\hline París'00 & 3 & 1 & 1 & & 2 & 14 \\
\hline \multicolumn{7}{|l|}{ San Luis'04 } \\
\hline \multicolumn{7}{|l|}{ Londres'08 } \\
\hline \multicolumn{7}{|l|}{ Estocolmo'12 } \\
\hline Amberes'20 & 56 & & 2 & & 2 & 17 \\
\hline París'24 & 102 & & & & & 28 \\
\hline Amsterdam'28 & 81 & 1 & & & 1 & 24 \\
\hline Los Ángeles'32 & 6 & & & 1 & 1 & 26 \\
\hline \multicolumn{7}{|l|}{ Berlín’36 } \\
\hline Londres'48 & 63 & & 1 & & 1 & 28 \\
\hline Helsinki'52 & 27 & & 1 & & 1 & 34 \\
\hline \multicolumn{7}{|l|}{ Melbourne'56 } \\
\hline Roma'60 & 145 & & & 1 & 1 & 41 \\
\hline Tokio'64 & 50 & & & & & 42 \\
\hline México'68 & 103 & & & & & 45 \\
\hline Munich'72 & 123 & & & 1 & 1 & 43 \\
\hline Montreal'76 & 114 & & 2 & & 2 & 30 \\
\hline Moscú'80 & 156 & 1 & 3 & 2 & 6 & 20 \\
\hline Los Ángeles'84 & 180 & 1 & 2 & 2 & 5 & 20 \\
\hline Seúl'88 & 216 & 1 & 1 & 2 & 4 & 25 \\
\hline Total & 1425 & 5 & 13 & 9 & 27 & \\
\hline Barcelona'92 & 421 & 13 & 7 & 2 & 22 & 6 \\
\hline Atlanta'96 & 289 & 5 & 6 & 6 & 17 & 13 \\
\hline Sídney’00 & 323 & 3 & 3 & 5 & 11 & 25 \\
\hline Atenas'04 & 316 & 3 & 11 & 6 & 20 & 20 \\
\hline Pekín’08 & 285 & 5 & 11 & 3 & 19 & 14 \\
\hline Londres'12 & 281 & 3 & 10 & 4 & 17 & 21 \\
\hline Rio'16 & 309 & 7 & 4 & 6 & 17 & 14 \\
\hline Total: & 2.224 & 39 & 52 & 32 & 123 & \\
\hline
\end{tabular}

De esas 27 medallas, sólo 5 fueron de oro (11,3\% del total). La obtención de oros es importante, no sólo por el hecho evidente de convertir en campeón olímpico al quien lo consigue, sino porque es uno de los principales criterios para establecer la posición de los países en el medallero final. 
Diplomas olímpicos (finalistas olímpicos)

La mejora que muestra España en el panorama Olímpico de las últimas décadas no se circunscribe al número de participantes o de preseas. Desde los Juegos de Londres 1948 los deportistas que ocupaban los puestos cuarto, quinto y sexto lugar en sus respectivas disciplinas han recibido certificados, oficialmente conocidos como Diplomas Olímpicos. En 1984, se extendió el número de Diplomas para los deportistas que ocuparon los puestos séptimo y octavo.

También el número de diplomas conseguidos presenta un cambio determinante en la participación olímpica española. De los 374 diplomas conseguidos a lo largo de la historia 270 (72.2\% - 7 ediciones de JJOO) pertenecen a la etapa posterior a Barcelona y sólo 104 a la etapa anterior (27.8\% - 21 ediciones de JJOO).

Si sumamos las preseas con los diplomas, vemos que el promedio de deportistas antes de Barcelona 92 era de 178 (8.0 deportistas por Medalla y Finalista) frente a 497 atletas (4.5 deportistas por Medalla y Finalista) de los últimos siete Juegos. Esto supone un cambio excepcional de rentabilidad costo/beneficie en el que, sin duda, el Plan ADO tiene un peso determinante (Tabla 9). 
Tabla 9. Diplomas obtenidos por el equipo olímpico español

\begin{tabular}{|c|c|c|c|c|c|c|}
\hline & $\begin{array}{l}4^{\circ} \\
\text { Pues } \\
\text { to }\end{array}$ & $\begin{array}{l}5^{\circ} \\
\text { Puesto }\end{array}$ & $\begin{array}{l}6^{\circ} \\
\text { Puesto }\end{array}$ & $\begin{array}{l}7^{\circ} \\
\text { Puesto }\end{array}$ & $\begin{array}{l}8^{\circ} \\
\text { Puesto }\end{array}$ & $\begin{array}{l}\text { Tota } \\
\text { I }\end{array}$ \\
\hline $\begin{array}{l}\text { Atenas'96 } \\
\text { París'00 } \\
\text { San Luis'04 } \\
\text { Londres'08 } \\
\text { Estocolmo'12 } \\
\text { Amberes'20 } \\
\text { París'24 } \\
\text { Amsterdam'28 } \\
\text { Los Ángeles'32 } \\
\text { Berlín'36 } \\
\text { Londres'48 } \\
\text { Helsinki'52 } \\
\text { Melbourne'56 } \\
\text { Roma'60 } \\
\text { Tokio'64 } \\
\text { México'68 } \\
\text { Munich'72 } \\
\text { Montreal'76 } \\
\text { Moscú'80 } \\
\text { Los Ángeles'84 } \\
\text { Seúl'88 } \\
\text { Barcelona'92 } \\
\text { Atlanta'96 } \\
\text { Sídney'00 } \\
\text { Atenas'04 } \\
\text { Pekín'08 } \\
\text { Londres'12 } \\
\text { Rio'16 }\end{array}$ & $\begin{array}{l}1 \\
2 \\
4 \\
3 \\
2 \\
5 \\
4 \\
10 \\
6 \\
5 \\
9 \\
6\end{array}$ & $\begin{array}{l}1 \\
1 \\
2 \\
5 \\
1 \\
4 \\
9 \\
9 \\
12 \\
14 \\
12 \\
6 \\
15\end{array}$ & $\begin{array}{l}1 \\
1 \\
1 \\
3 \\
3 \\
8 \\
1 \\
6 \\
8 \\
7 \\
9 \\
5 \\
7 \\
4\end{array}$ & $\begin{array}{l}7 \\
4 \\
9 \\
10 \\
9 \\
14 \\
7 \\
5 \\
7\end{array}$ & $\begin{array}{l}3 \\
2 \\
7 \\
4 \\
6 \\
8 \\
6 \\
4 \\
7\end{array}$ & $\begin{array}{l}1 \\
2 \\
2 \\
2 \\
7 \\
12 \\
22 \\
13 \\
36 \\
35 \\
44 \\
51 \\
35 \\
31 \\
39\end{array}$ \\
\hline Total: & 59 & 95 & 65 & 72 & 47 & 338 \\
\hline
\end{tabular}

Fuente: elaboración propia

El Programa ADO en el periodo 2005-2016

Aunque el Programa ADO se inicia en el ciclo olímpico que va desde 1988 a 1992, en este trabajo nos vamos a centrar únicamente en el periodo que va desde 2005 a 2016, es decir el periodo que incluye las Olimpiadas de Pekín, Londres y Rio de Janeiro.

Según datos del Comité Olímpico Español, en este periodo se han ganado 54 medallas (Hombres: 28 medallas - Mujeres: 26 mujeres) y 104 Diplomas (Hombres: 62 Diplomas - Mujeres: 42 Diplomas) (Tabla 10). Realmente interesante supone la importante progresión de los atletas de la categoría femenina. 
Tabla 10. Evolución de los medallistas olímpicos españoles en los JJOO (1992-2016)

\begin{tabular}{|c|c|c|c|c|}
\hline \multirow{2}{*}{$\begin{array}{l}\text { JJOO } \\
\text { AÑO }\end{array}$} & \multicolumn{4}{|c|}{ MEDALLAS OLÍMPICAS } \\
\hline & TOTAL & HOMBRES & MUJERES & $\% \mathrm{H}-\mathrm{M}$ \\
\hline 1992 & 22 & 14 & 8 & 36,4 \\
\hline 1996 & 17 & 11 & 6 & 35,3 \\
\hline 2000 & 11 & 7 & 4 & 36,4 \\
\hline 2004 & 20 & 14 & 6 & 30,0 \\
\hline 2008 & 19 & 14 & 5 & 26,3 \\
\hline 2012 & 18 & 6 & 12 & 66,7 \\
\hline 2016 & 17 & 8 & 9 & 52,9 \\
\hline JJOO & \multicolumn{4}{|c|}{ DIPLOMAS - PUESTOS $4^{\circ}$ A $8^{\circ}$} \\
\hline AÑO & TOTAL & HOMBRES & MUJERES & $\%$ H-M \\
\hline 1992 & 36 & 25 & 11 & 30,6 \\
\hline 1996 & 35 & 25 & 10 & 28,6 \\
\hline 2000 & 44 & 24 & 20 & 45,5 \\
\hline 2004 & 51 & 26 & 25 & 49,0 \\
\hline 2008 & 35 & 21 & 14 & 40,0 \\
\hline 2012 & 30 & 21 & 9 & 30,0 \\
\hline 2016 & 39 & 20 & 19 & 48,7 \\
\hline
\end{tabular}

Aportación económica global e inversión en becas

Desde su creación, el Plan ADO ha contado con 335.5 millones de euros, de los cuales 137 millones corresponden al periodo objeto de estudio (2005-2016). Una parte de esta cantidad se dedica a gastos de gestión, promoción y representación, aunque la mayor parte se invierte en las Becas de ayuda a los deportistas. La inversión en Becas fue de 81.628.774 de euros, de los que 46.697.523 euros se invirtieron en deportistas de la categoría masculina y 34.918.874 euros en la categoría femenina (Tabla 11). Este dinero es proporcionado por Socios (Coca-Cola, La Caixa, Estrella Damm, Danone, El Corte Inglés, Repsol, Telefónica y Loterías y Apuestas del Estado) y Empresas patrocinadoras (Allianz, Pascual Cola Cao, Correos y Barceló Viajes). Desde un punto de vista de la rentabilidad deportiva del Plan ADO, el precio de cada medalla fue de 1.511.644 euros (Hombres: 1.667 .769 euros; Mujeres: 1.343.034 euros).

Es un hecho que la crisis económica de 2008 tuvo un claro reflejo en los recursos disponibles para sostener el deporte de alto nivel, especialmente, entre los deportes menos profesionalizados. Aunque se considera que la crisis surgió en 2008, especialmente tras la caída del banco estadounidense Lehman Brothers, los primeros síntomas aparecieron en agosto de 2007 con la quiebra de varios bancos de inversión de menor importancia. Esto obligó a los gobiernos, especialmente los de los países más desarrollados, a realizar numerosos rescates financieros de una eminente quiebra, que junto a la fuerte caída de los ingresos en la recaudación fiscal, hizo que estos países se vieran 
abocados a la implantación de severos programas de austeridad económica que implicaban fuertes recortes sociales y de inversión pública.

Tabla 11. Evolución de la inversión en becas olímpicas españolas (2005-2016)

\begin{tabular}{|l|l|l|l|l|}
\hline AÑO & $\begin{array}{l}\text { Inversión Total } \\
\text { (millones) }\end{array}$ & $\begin{array}{l}\text { Inversión Hombres } \\
\text { (millones) }\end{array}$ & $\begin{array}{l}\text { Inversión Mujeres } \\
\text { (millones) }\end{array}$ & $\begin{array}{l}\text { Ratio } \\
\text { H:M }\end{array}$ \\
\hline 2005 & 7.414 .030 & 4.441 .625 & 2.980 .400 & 1,49 \\
2006 & 8.713 .656 & 5.529 .750 & 3.182 .400 & 1,74 \\
2007 & 7.971 .714 & 4.816 .707 & 3.153 .500 & 1,53 \\
2008 & 6.810 .183 & 3.956 .125 & 2.852 .300 & 1,39 \\
2009 & 6.839 .209 & 3.914 .500 & 2.923 .200 & 1,34 \\
2010 & 7.282 .660 & 4.163 .875 & 3.116 .775 & 1,34 \\
2011 & 7.460 .536 & 4.257 .175 & 3.201 .350 & 1,33 \\
2012 & 6.094 .262 & 3.909 .500 & 2.182 .750 & 1,79 \\
2013 & 3.748 .365 & 2.007 .752 & 1.738 .600 & 1,15 \\
2014 & 6.476 .654 & 3.086 .700 & 3.387 .940 & 0,91 \\
2015 & 6.705 .239 & 3.433 .614 & 3.269 .609 & 1,05 \\
2016 & 6.112 .266 & 3.180 .200 & 2.930 .050 & 1,09 \\
\hline
\end{tabular}

Fuente: elaboración propia

La tabla 11 muestra como hay una disminución presupuestaria notable durante los últimos años, que en parte es debida a la diferencia de modalidades deportivas que se van incluyendo cada año en cada presupuesto. Especialmente bajo es el volumen económico de Becas de la temporada 2013, donde no se alcanzan lo 4 millones de euros y donde 9 federaciones no recibieron ayudas (Bádminton, Boxeo, Esgrima, Fútbol, Golf, Pentatlón Moderno, Remo, Rugby y Tiro con Arco). Para las Olimpiadas de Pekín, Londres y Rio Janeiro el programa contó con 147.5 millones de Euros, con una caída significativa y vertiginosa en esos ciclos olímpicos: Se pasa de 30.909 .583 millones para Pekín, a 27.676.667 millones para Londres (descenso del 10.5\%) y a 19.668.994 millones para los pasados Juegos de Rio (descenso del 31.4\%).

Pese a esta importante caída económica, la rentabilidad deportiva no se ha visto seriamente mermada. El coste de las medallas bajó a 1.537 .593 millones en Londres frente al 1.626.820 millones de Pekín, y a 1.157.000 millones en Rio. Es decir, estamos hablando de una reducción del $28.9 \%$ en el coste de cada presea.

Más interesante aún es lo que ocurre con las mujeres. Mientras en Pekín el coste de la medalla fue 2.433.760 millones, pasa a 1.904 .013 millones en Londres (descenso del $21.8 \%$ ) y a 1.258 .467 millones (un descenso del $48.3 \%$ ) en los últimos Juegos analizados (Río 16). Esto es una clara muestra del auge del deporte femenino español y del desarrollo que muestra en el número de deportistas y la calidad de su rendimiento.

Hasta hace pocas décadas, generalmente los hombres han estado más involucrados que las mujeres en la práctica deportiva (Guttmann, 1986). Los trabajos orientados a evaluar la relación de hombres y mujeres involucrados en el deporte han sido numerosos en los últimos años (Stamatakis y Chaudhury, 2008; Ferrar, Olds y Walters, 2012; Deaner y Smith, 2013). Todos ellos coinciden en señalar que existe una diferencia entre sexos que es clara y consistente. 
Estos estudios informan que los hombres practican deportes con mayor frecuencia que las mujeres, generalmente al menos el doble en términos de duración o frecuencia (Deaner et al., 2012). Sin embargo, estos datos parecen estar invirtiéndose entre las nuevas generaciones y, de forma muy destaca, entre los jóvenes españoles que, quizás no aumente tanto su nivel de práctica deportiva, sino que han mejorado sustancialmente en su calidad y competitividad hasta convertirse muchas deportistas españolas en verdaderos iconos sociales.

\section{CONCLUSIONES}

Diferentes autores plantean que, aunque un número creciente de naciones invierte importantes cantidades de dinero en el deporte de alto nivel para afrontar con garantías de éxito las principales competiciones internacionales, no hay evidencias claras que demuestren y expliquen cómo las políticas deportivas gubernamentales pueden influir en el éxito deportivo internacional. No obstante, crear un sistema de racionalización de las inversiones podría ser un factor potenciador de las posibilidades iniciales de obtener unos buenos resultados. Si bien un sistema deportivo nacional es algo complejo, que afecta de forma diferente a la sociedad y un alto número de sus organismos e instituciones públicas, pocos pueden poner en duda que el cuidado especial de los deportistas más destacados, y de los equipos técnicos encargados de su preparación, es uno de los aspectos más importantes.

En el caso de España, sin duda, y a la luz de los datos mostrados, el Plan ADO ha sido uno de los factores claves de la transformación deportiva en España y de sus éxitos deportivos en la esfera internacional desde los JJOO de Barcelona 92, contribuyendo a potenciar y a afianzar el deporte olímpico español. En este trabajo hemos podido comprobar como, desde la instauración de este programa, el deporte de alto nivel español ha dado un salto hacia delante en términos cuantitativos y cualitativos. Desde su hasta la edición de Rio de Janeiro, se ha logrado clasificar el $61 \%$ del total histórico de los atletas olímpicos españoles, se han obtenido el $82 \%$ de las medallas y el $72 \%$ de los diplomas. Estos datos demuestran la relación directa existente entre inversión económica en el deporte de alto nivel y los éxitos cosechados en las citas olímpicas.

Aunque hemos podido comprobar como el Plan ADO ha ido sufriendo oscilaciones en su financiación, especialmente por su dependencia parcial de las inversiones de empresas privadas, que han decrecido de manera significativa desde 2008 , lo cierto es que los datos de participación no se han visto seriamente significativamente afectados.

Es un hecho que, desde su creación, las becas ADO han sido obtenidas de forma mayoritaria por hombres. No obstante, en los dos últimos ciclos olímpicos hemos podido observar como el número de becas y la cuantía total de las mismas, ha ido creciendo en favor de las mujeres y, en algunos años concretos incluso han sido superior que las masculinas. Ello ha coincido con el hecho histórico de que en los JJOO de Londres y Rio las mujeres españolas superaron por primera vez a los hombres en medallas obtenidas. Estos datos denotan que el incremento de las ayudas al deporte femenino en el Plan ADO podría explicar la mejora reciente de los resultados de las deportistas españolas, y que existe una mayor 
rentabilidad en cuanto a la inversión en becas en las mujeres respecto de los hombres

\section{REFERENCIAS BIBLIOGRÁFICAS}

Barker-Ruchti, N., Schubring, A., Aarresola, O., Kerr, R., Grahn, K., y McMahon, J. (2018). Producing success: A critical analysis of athlete development governance in six countries. International Journal of Sport Policy and Politics, 10(2), 215-234. https://doi.org/10.1080/19406940.2017.1348381

Bergsgard, N.A., Houlihan, B., Mangset, P., Nodland, S.I., y Rommetveldt, H. (2007). Sport policy. A comparative analysis of stability and change. London: Elsevier.

Bernard, A.B. y Meghan R. B. (2004). 'Who Wins the Olympic Games: Economic Resources and Medal Totals', Review of Economics and Statistics, 86(1), 413417. https://doi.org/10.1162/003465304774201824

Calle-Molina, M.T. y Martínez-Gorroño, M.E. (2019). José Enrique Cal: primer medallista español de boxeo olímpico. Revista Internacional de Medicina y Ciencias de la Actividad Física y el Deporte, 19(73), 77-92. http://doi.org/10.15366/rimcafd2019.73.006

Castejón, B, de Dios-García, J., y Carballada, R, (1973). Rationalising Sports Policies: I. Outline of a Methodology. European Cooperation for the Development of Sport for All. Strasburg: Concil of Europe / Comittee for Out of School Education y Cultural Development.

Conzelmann, A., y Nagel, S. (2003). Professional careers of the German Olympic athletes. International Review for the Sociology of Sport, 38, 259-280. https://doi.org/10.1177/10126902030383001

De Bosscher, V., De Knop,P., van Bottenburg, M. y Shibli, S. (2006). A conceptual framework for analysing sports policy factors Leading to international sporting success. European Sport Management Quarterly, 6(2), 185-215. https://doi.org/10.1080/16184740600955087

De Bosscher, V., Bingham, J., Shibili, S., Van Botenburg, M. y De Knop, P. (2008). The global sporting arms race: An international comparative study on sports policy factors leading to international sporting success. Oxford: Meyer y Meyer Sport.

De Bosscher, V., De Knop, P., Van Bottenburg, M., Shibli, S., y Bingham, J. (2009). Explaining international sporting success: An international comparison of elite sport systems and policies in six countries. Sport Management Review, 12(3), 113-136. https://doi.org/10.1016/j.smr.2009.01.001

De Bosscher, V., Shibli, S., Westerbeek, H., y Van Bottenburg, M. (2015). Successful elite sport policies: an international comparison of the sports policy factors leading to international sporting success (SPLISS 2.0) in 15 nations. Oxford: Meyer y Meyer Sport.

Deaner, R. O., Geary, D. C., Puts, D. A., Ham, S. A., Kruger, J., Fles, E., y Grandis, T. (2012). A sex difference in the predisposition for physical competition: Males play sports much more than females even in the contemporary U.S. PLOS ONE, 7(11). http://dx.doi.org/10.1371/journal.pone .0049168 . 
Deaner, R. O. (2013). Distance running as an ideal domain for showing a sex difference in competitiveness. Archives of Sexual Behavior, 42, 413-428. https://doi.org/10.1007/s10508-012-9965-z

Deaner, R. O., Balish, S. M., y Lombardo, M. P. (2016). Sex differences in sports interest and motivation: An evolutionary perspective. Evolutionary Behavioral Sciences, 10(2), 73-97. https://doi.org/10.1037/ebs0000049

Douyin, X. (1988). A comparative study on the competitive sports training systems in different countries. Journal of Comparative Physical Education and Sport, 2(3), 3-12.

Dyer, J. (2005). High performance sport menagement. In Lebermann, S., Trenberth,L., y Collins, C. (Eds), En Sport Business Management in New Zealand. Auckland : Dunmore Press.

Ferrar, K. E., Olds, T. S., y Walters, J. L. (2012). All the stereotypes confirmed: Differences in how Australian boys and girls use their time. Health Education y Behavior, 39, 589 -595. https://doi.org/10.1177/1090198111423942

Funahashi, H., De Bosscher, V., y Mano, Y. (2015). Understanding public acceptance of elite sport policy in Japan: a structural equation modelling approach. European Sport Management Quarterly, 15(4), 478-504. https://doi.org/10.1080/16184742.2015.1056200

Gibbons, T., McConnel, A., Forster, T., Riewald, ST. y Peterson, K. (2003). Reflections on success: US Olympians describe the Success Factors and obstacles that most influenced their Olympic development. Report phase II, United States Olympic Committee (USOC).

Gómez, S., Martí, C., Gigante, J., y Opazo, M. (2011). El plan ADO desde la perspectiva de deportistas, patrocinadores e institucionalidad: una evaluación basada en el dialogo entre los agentes. Documento de Investigación DI-926. IESE Business School - Universidad de Navarra.

Green, M., y Houlihan, B. (2005). Elite sport development. Policy learning and political priorities. London and New York: Routledge.

Greenleaf, C., Gould, D., y Diefen, K. (2001). Factors influencing Olympic performance with Atlanta and Nagano US Olympians. Journal of applied sport psychology, 13, 154- 184. https://doi.org/10.1080/104132001753149874

Guttmann, A. (1986). Sports spectators. New York, NY: Columbia University Press.

Haut, J., Grix, J., Brannagan, P. M., y Hilvoorde, I. V. (2017). International prestige through 'sporting success': an evaluation of the evidence. European Journal for Sport and Society, 14(4), 311-326. https://doi.org/10.1080/16138171.2017.1421502

Houlihan, B., y Green, M. (2008). Comparative elite sport development. Systems, structures and public policy. London: Elsevier.

Humphreys, B. R., Johnson, B. K., Mason, D. S., y Whitehead, J. C. (2018). Estimating the Value of Medal Success in the Olympic Games. Journal of Sports Economics, 19(3), 398-416. https://doi.org/10.1177/1527002515626221

Johnson, D., y Ali, A. (2004). A Tale of Two Seasons: Participation and Medal Counts at the Summer and Winter Olympic Games. Social Science Quarterly, 85(4), 974-993. https://doi.org/10.1111/j.0038-4941.2004.00254.x 
Jones, M. B. (2013). The home advantage in individual sports: An augmented review. Psychology of Sport and Exercise, 14(3), 397-404. https://doi.org/10.1016/j.psychsport.2013.01.002

Kiviaho, P., y Mäkelä, P (1978). Olympic Success: A sum of non-material and material factors. International Review of Sport Sociology, 2, 5-17. https://doi.org/10.1177/101269027801300201

Krishna, A., y Haglund, E. (2008). Why do some countries win more Olympic medals? Lessons for social mobility and poverty reduction. Economic and Political Weekly, 43, 143-151.

Krüger, A. (1984). To Moscow and back: international status of comparative research in regard to physical activity outside of schools. Actas del 4th International Seminar on Comparative Physical Education and Sport. MalenteKiel, Alemania Occidental, 213-227.

Kuettel, A., Boyle, E., y Schmid, J. (2017). Factors contributing to the quality of the transition out of elite sports in Swiss, Danish, and Polish athletes. Psychology of sport and exercise, 29, 27-39. https://doi.org/10.1016/j.psychsport.2016.11.008

Leiva-Arcas, A. y Sánchez-Pato, A. (2019). Análisis de los resultados de España en su participación en los Juegos Olímpicos de verano. En Alberto Pérez y Julio Pernas (Eds.), El olimpismo en España. Una mirada histórica de los orígenes a la actualidad (pp. 295-336). Barcelona: Fundación Barcelona Olímpica.

Legaz-Arrese, A., Moliner-Urdiales, D., y Munguía-Izquierdo, D. (2013). Home advantage and sports performance: evidence, causes and psychological implications. Universitas Psychologica, 12(3), 933-943. https://doi.org/10.11144/Javeriana.UPSY12-3.hasp

Morton, R.H. (2002). Who won the Sydney 2000 Olympics? An allometric approach. The Statistician, 51, 147-155. https://doi.org/10.1111/14679884.00307

Pérez-Aragón, P. y Gallardo-Pérez, J. (2017). Coubertin y los concursos artísticos en los Juegos Olímpicos modernos. Revista Internacional de Medicina y Ciencias de la Actividad Física y el Deporte, 17(68), 633-649. https://doi.org/10.15366/rimcafd2017.68.004

Puig, N., Martínez, J., y García, B. (2010). Sport policy in Spain. International Journal of Sport Policy, 2(3), 381-390. https://doi.org/10.1080/19406940.2010.519343

Reiche, D. (2016). Success and failure of countries at the Olympic Games. London: Routledge.

Riordan, J. (1989). Soviet Sport and Perestroika. Journal of Comparative Physical Education and Sport, 6, 7-18.

Robles-Rodríguez, A., Abad-Robles, M.T., Robles-Rodríguez, J. y GiménezFuentes, F.J. (2019). Factores que influyen en el proceso de formación de los judokas olímpicos. Revista Internacional de Medicina y Ciencias de la Actividad Física y el Deporte, 19(74), 259-276. http://doi.org/10.15366/rimcafd2019.74.006

Santacama, C. (2011). Espejo de un régimen. Transformación de las estructuras deportivas y su uso politico y propagandístico, 1939-1961. En Xavier Pujadas (coord.), Atletas y ciudadanos. Historia social del deporte en España, 18702010 (pp. 205-232). Madrid: Alianza Editorial. 
Semotiuk, D. M. (1990). East Bloc Athletics in the Glasnost Era. Journal of Comparative Physical Education and Sport, 9(1), 26-29.

Sergeyev, Y.D. (2015). The olympic medals ranks, lexicographic ordering and numerical infinities. The Mathematical Intelligencer, 37(2), 4-8. http://doi.org/10.1007/s00283-014-9511-z

Stamatakis, E., y Chaudhury, M. (2008). Temporal trends in adults' sports participation patterns in England between 1997 and 2006: The Health Survey for England. British Journal of Sports Medicine, 42, 901-908. http://dx.doi.org/10.1136/bjsm.2008.048082

Número de citas totales / Total references: 43 (100\%)

Número de citas propias de la revista /Journal's own references: 3 (6,97\%)

Rev.int.med.cienc.act.fís.deporte - vol. 21 - número 83 - ISSN: 1577-0354 